\title{
Long-term potentiation in frontal cortex: role of NMDA-modulated polysynaptic excitatory pathways
}

\author{
Bernd Sutor* and John J. Hablitz \\ Section of Neurophysiology, Department of Neurology, Baylor College of Medicine, Houston, TX 77030 \\ (U.S.A.)
}

(Received 6 September 1988; Revised version received 3 October 1988; Accepted 4 October 1988)

Key words: Neocortex; Long-term potentiation; $N$-Methyl-D-aspartic acid receptor; Excitatory postsynaptic potential

The present study examined the role of $N$-methyl-D-aspartic acid (NMDA) receptors in synaptic plasticity in regular-spiking cells of rat frontal cortex. Intracortical stimulation, at levels subthreshold for elicitation of action potentials, evoked a late excitatory postsynaptic potential (EPSP) in layer II III neurons that was sensitive to the selective NMDA antagonist D-2-amino-5-phosphonovaleric acid (APV). This late EPSP showed marked short-term frequency-dependent depression, suggesting that it is polysynaptic in origin. Polysynaptic late EPSPs were selectively enhanced following high-frequency stimulation. This sustained increase in synaptic efficacy, or long-term potentiation, was expressed in regular spiking cells and appeared to result from activation of NMDA receptors on excitatory interneurons. These data demonstrate the existence of an NMDA-modulated polysynaptic circuit in the neocortex which displays several types of use-dependent plasticity.

Neocortical neurons can display sustained increases in synaptic efficacy following conditioning stimulation $[2,3,5]$. The mechanisms underlying this long-term potentiation (LTP) are unclear. Although many studies have indicated that LTP is due to an increased effectiveness of monosynaptic connections [1, 11], the possibility that alterations in the activity of excitatory local circuit neurons can also underlie changes in synaptic efficacy has been raised [20]. Recent interest in neural networks as devices for information storage and retrieval $[14,19]$ suggests that interactions between local mosaics of neurons may be important in learning and memory as well as changes in monosynaptic connections. This is especially relevant in the neocortex where $70 \%$ of fibers forming asymmetric synapses arise from intrinsic neurons involved in local excitatory circuits [15]. In examining synaptic plasticity in the neocortex, we have found a type of LTP that involves selective enhancement of polysynaptic EPSPs.

\footnotetext{
*Present address: Physiologisches Institut der Universität, Pettenkoferstrasse 12, 8000 München 2, F.R.G. Correspondence: J.J. Hablitz, Neurobiology Research Center, Department of Physiology and Biophysics, University of Alabama at Birmingham, Birmingham, AL 35294, U.S.A.
} 
Brain slices from the frontal cortex of adult rats were prepared and maintained in vitro as described previously $[9,16]$. Intracellular recordings were obtained from layer II--III neurons which were classified as regular-spiking neurons [12]. A bipolar stimulating electrode (platinum iridium wire $30 \mu \mathrm{m}$ diameter) was positioned in layer IV and used to deliver stimuli $50-200 \mu \mathrm{s}$ in duration. The mean resting membrane potential (RMP) and mean input resistance of these neurons were $>-75 \mathrm{mV}$ and $>18 \mathrm{M} \Omega$, respectively. Action potential amplitudes were $>100 \mathrm{mV}$. Intracortical electrical stimulation in layers IV-- $V$ with very low intensities $(0.1-0.35 \mathrm{~T}$, where $\mathrm{T}$ is the threshold for action potential elicitation) elicited an EPSP at a latency of 2--3 ms. This EPSP had an amplitude of $49 \mathrm{mV}$, a time to peak of $413 \mathrm{~ms}$, and a duration of $3066 \mathrm{~ms}$ (Fig. 1A). Depolarization of the RMP by $10-20 \mathrm{mV}$ and measurements of input resistance revealed no sign of an inhibitory postsynaptic potential (IPSP). Increases in stimulus intensity (to values between 0.4 and $0.5 \mathrm{~T}$ ) produced an increase in EPSP amplitude and the appearance of a second depolarizing potential (Fig. 1A, middle trace) with an amplitude of $10-40 \mathrm{mV}$, a time to peak of 25-65 ms, and a duration of $80-165 \mathrm{~ms}$. Incrementing the stimulus intensity produced an initial increase in amplitude and a decrease in latency of the second response. The long and variable latency of this second response suggests that it is polysynaptic in origin (see

A

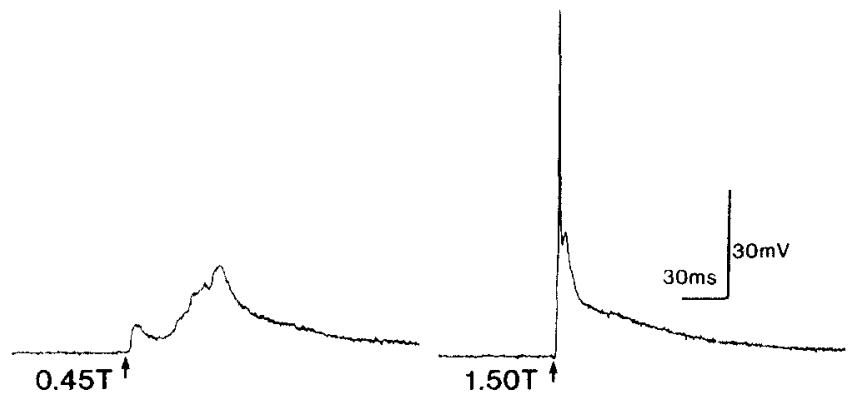

B

control

D-2-APV, 20pM, 4min

wash, $\left.18 \mathrm{~min} 30 \mathrm{~ms}\right|^{20 \mathrm{mV}}$
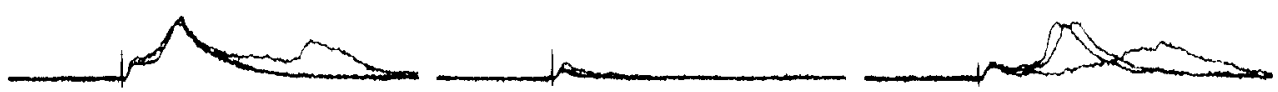

Fig. 1. Weak intracortical stimulation evokes an APV-sensitive l-EPSP. A: intracellular recordings of synaptic potentials evoked by stimuli of increasing intensities. Left trace, a $0.30 \mathrm{~T}$ stimulus (where $\mathrm{T}$ is threshold for eliciting an action potential) evokes an e-EPSP; middle trace, increasing stimulation to 0.45 $\mathrm{T}$ produces an l-EPSP; right trace, a high stimulus strength (1.5 $\mathrm{T}$ ) evokes an action potential followed by a depolarizing IPSP which suppresses the l-EPSP. Time of stimulation is indicated by arrows. Resting membrane potential (RMP) was $-85 \mathrm{mV}$. B: the l-EPSP was blocked by APV. Left panel, this neuron $(\mathrm{RMP}=-79 \mathrm{mV})$ responded to electrical stimulation $(0.50 \mathrm{~T})$ with an e-EPSP followed by an 1-EPSP (control). Middle panel, upon addition of APV $(20 \mu \mathrm{M})$ to the bathing solution, the l-EPSP was abolished, and the e-EPSP was reduced. Right panel, the effects of APV were reversible (wash, 18 min). APV influenced neither RMP nor the current-voltage relation of the neuron. Each panel consists of 3 consecutive traces superimposed. 
below). Following depolarization of the RMP by $10-15 \mathrm{mV}$, this second response was capable of generating action potentials, suggesting that it was an EPSP. Again. there was no evidence that stimulation at this intensity evoked an IPSP. This second response was therefore considered to be an EPSP, and, using the time to peak as criterion, we defined the initial small EPSP as an early EPSP (e-EPSP) and the second response as a late EPSP (l-EPSP). Late EPSPs were seen in $>95 \%$ of neurons tested $(n=55)$. When stimulus intensities greater than $0.75 \mathrm{~T}$ were used, l-EPSPs were paradoxically smaller in amplitude or absent. We attribute this to the elicitation of IPSPS at these stimulus strengths (Fig. 1A, rightmost trace). During the IPSP, which is depolarizing at rest $[9,18]$, the neuronal input resistance is markedly reduced. Due to the shunting effect of the chloride-dependent IPSP [18], the I-EPSP is not seen. The observation that the l-EPSP was depressed by IPSPs following stimulation with intensities larger than $0.75 \mathrm{~T}$ was made in all neurons in which a complete inputoutput relationship was determined ( $n=35$; see Fig. $3 \mathrm{C}$ ). These results demonstrate that the l-EPSP is observed only over a certain range of stimulus strengths.

As shown in Fig. 1B, upon addition of the NMDA receptor antagonist APV (5-20 $\mu \mathrm{M})$ to the bathing solution, the l-EPSP was found to be selectively and reversibly abolished in all neurons tested $(n=10)$, suggesting that NMDA receptors were involved in its generation. Similar APV concentrations selectively block responses to iontophoretically applied NMDA in neocortical neurons [10]. In contrast, the cEPSPs was insensitive to APV, even after depolarization of the membrane potential to just subthreshold levels. The apparent reduction seen in Fig. $1 \mathrm{~B}$ was due to a superimposition of the 1-EPSP on the e-EPSP in this cell. Pure e-EPSPs evoked by stimulation intensities subthreshold for 1-EPSPs were unaffected by APV (e-EPSP = $5.8 \pm 2.6$ and $6.2 \pm 2.3 \mathrm{mV}$ before and after APV, respectively; $n=6$ ).

The amplitude of the l-EPSP was readily influenced by changing the membrane potential using intracellular current injection (Fig. 2A). In the range investigated $(-110$ to $-50 \mathrm{mV})$, the amplitude of the 1-EPSP was a direct function of membrane potential, increasing with hyperpolarization and decreasing with depolarization. This indicates that the 1-EPSP is likely to be a synaptic potential generated at a site influenced by somatic current passage and not the result of active dendritic responses. The observation of a conventional voltage sensitivity for the APV-sensitive 1-EPSP was, however, unexpected and in contrast to previous studies of EPSPs [17] and NMDA responses [8] in neocortical neurons. We therefore directly compared the voltage sensitivity of the 1-EPSP and NMDA responses. In Fig. 2B, the iontophoretic current used to apply NMDA was adjusted to evoke, at the RMP, a slow depolarization that produced a burst of action potentials (Fig. 2B. middle trace). Upon hyperpolarization (Fig. 2B, leftmost trace), the depolarization was reduced and the bursting abolished. When depolarized (Fig. 2B, last trace), this neuron responded to NMDA with an enhanced depolarization and increased frequency of bursting. Simultaneously, the l-EPSP, seen at the beginning of each trace, increased with hyperpolarization and decreased with depolarization. This discrepancy between the voltage dependence of the l-EPSP and that of the NMDA-induced responses suggests that NMDA receptors located on the impaled neurons are not involved in the gener- 
A Stimulus: 0.3T
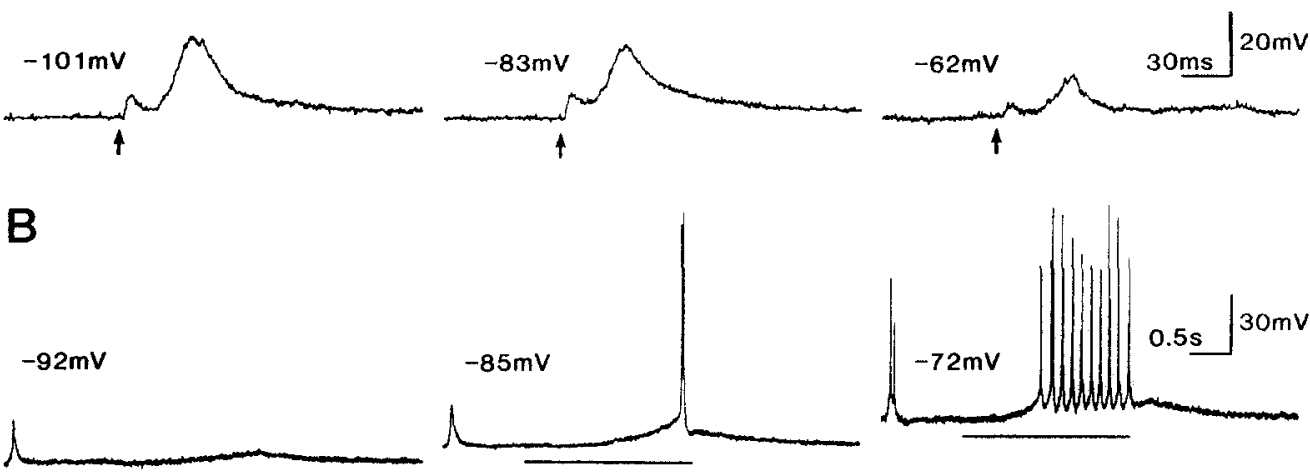

$\overline{\text { NMDA 50nA }}$

Fig. 2. Voltage dependence of the 1-EPSP and NMDA responses. A: synaptic response of a neuron $(R M P=-83 \mathrm{mV})$ to electrical stimulation $(0.3 \mathrm{~T})$ at different membrane potentials. Note the decrease in the e-EPSP amplitude with hyperpolarization (due to the activation of hyperpolarizing inward rectification). B: examples of NMDA-induced depolarizations at different membrane potentials in a different neuron $(\mathrm{RMP}=-85 \mathrm{mV}$ ). NMDA was applied by iontophoresis with $50 \mathrm{nA}$ for $2 \mathrm{~s}$ (indicated by the bars below the traces). The voltage deviation at the beginning of each trace represents the stimulus-evoked 1 EPSP. Note the action potentials generated by the 1 -EPSP at $-72 \mathrm{mV}$. Action potentials are truncated.

ation of the l-EPSP. Consequently, we regard the observed APV sensitivity of the 1-EPSP as an effect on NMDA receptors located on interneurons responsible for generating the 1-EPSP. We therefore tested whether the 1-EPSP was polysynaptic in origin by testing its ability to follow repetitive stimulation.

In the 5 neurons tested systematically, the l-EPSP failed at stimulation frequencies greater than $0.5 \mathrm{~Hz}$, whereas the e-EPSP was not significantly altered (Fig. 3A). In all cases, there was an abrupt decline of the 1-EPSP amplitude as the frequency of stimulation was increased. As shown in the middle trace of Fig. 3A, only the first stimulus at $1.0 \mathrm{~Hz}$ produced a clearly detectable l-EPSP. The succeeding nine stimuli evoked only an e-EPSP. The l-EPSP recovered completely within one stimulus cycle after the cessation of rapid stimulation. The precipitant suppression of the 1-EPSP by stimulation at frequencies as low as $1 \mathrm{~Hz}$ and its fast and complete recovery are indicative of involvement of a polysynaptic pathway in generating the l-EPSP [4].

A characteristic feature of the 1-EPSP was its ability to show long-term changes in excitatory efficacy. Following tetanization ( $1-4$ trains of $100 \mathrm{~Hz}$ for $1 \mathrm{~s}$, delivered at 5-s intervals), a long-lasting enhancement of excitatory synaptic transmission was observed in 14 of 16 neurons. As can be seen in Fig. 3B, the 1-EPSP was preferentially enhanced, increasing by $120-270 \%$. This is shown clearly in the rightmost trace of Fig. 3B which shows the difference between the responses to a $7.5 \mathrm{nC}$ test stimulus before and $15 \mathrm{~min}$ after the tetanus. The net effect in this cell was a selective increase in the 1-EPSP. Examination of input-output relations (Fig. 3C) revealed that significant enhancement of synaptic potentials was observed only with stimulation intensities below threshold for activation of inhibitory circuits $(20 \mathrm{nC}$ in this case). Beyond 
A

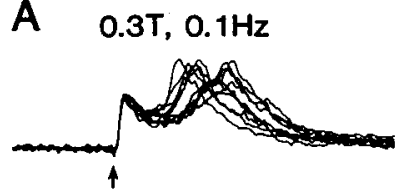

B

control
$0.3 \mathrm{~T}, 1.0 \mathrm{~Hz}$

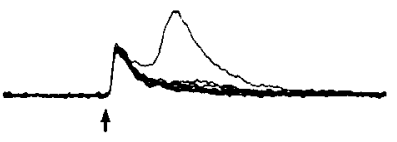

15 min after HFS

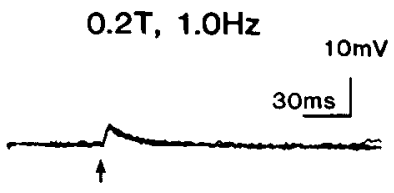

difference
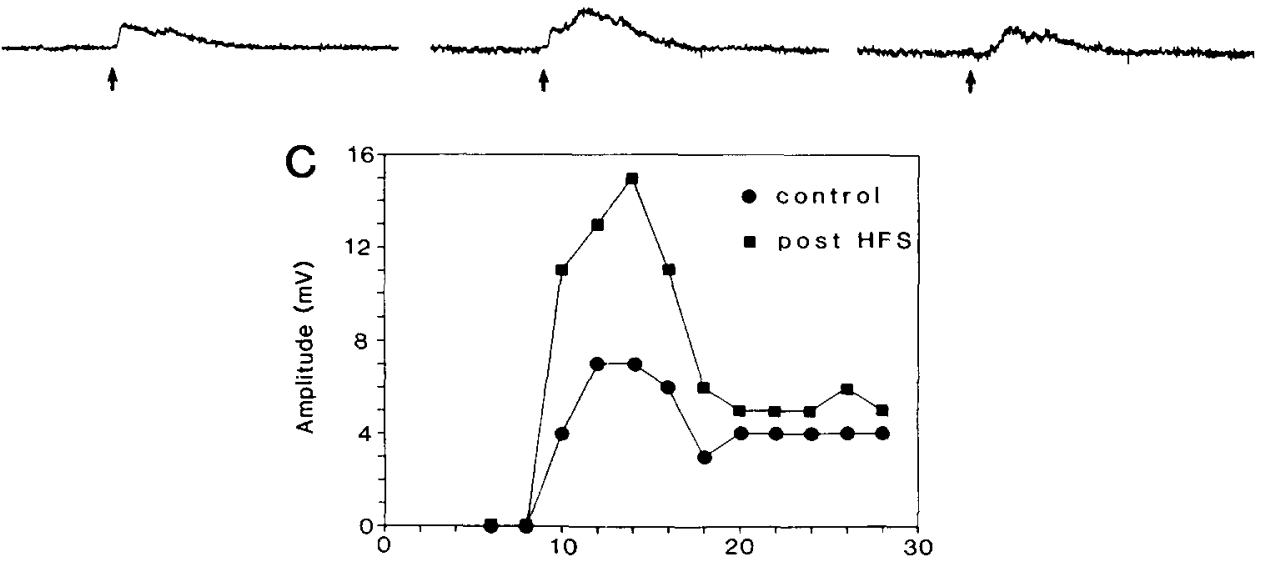

Fig. 3. Frequency-dependent short- and long-term changes in the 1-EPSP. A: depression of the 1-EPSP by stimulation at a frequency of $1 \mathrm{~Hz}$. The stimulus intensity was adjusted to evoke an e-EPSP followed by an l-EPSP $(0.3 \mathrm{~T}$ or $12.5 \mathrm{nC})$. Each trace represents 10 superimposed consecutive responses. The rightmost trace depicts 10 superimposed responses to stimulation at $1 \mathrm{~Hz}$ when the stimulus intensity was reduced to values subthreshold for the l-EPSP $(0.2 \mathrm{~T}$ or $10 \mathrm{nC})$. RMP $=-81 \mathrm{mV}$. B: LTP of the l-EPSP in another neocortical neuron (RMP $=-84 \mathrm{mV}$ ). Stimulation evoked an e-EPSP followed by a small 1 EPSP. Ten min after high-frequency stimulation (HFS), the same stimulus evoked an e-EPSP and an enhanced 1-EPSP. The rightmost trace is a digital subtraction of the first two traces and shows the difference between the recording before and after LTP. Each trace is an average of 10 single measurements performed at a frequency of $0.1 \mathrm{~Hz}$. Stimulation consisted of four trains of $100 \mathrm{~Hz}$ for $1 \mathrm{~s}$ delivered at $5 \mathrm{~s}$ intervals. Stimulus strength was at the threshold intensity necessary to evoke an e-EPSP. C: input output relationship determined before and 19 min after LTP. I-EPSP amplitudes were measured at their peaks. Each point is the response to a single stimulation. High-frequency stimulation consisted of a single I s train of $100 \mathrm{~Hz}$ stimulation at half of e-EPSP threshold.

these levels, IPSPs masked the effect of high-frequency stimulation in addition to shunting the 1-EPSP.

The induction of LTP in neocortical neurons was blocked by APV (5-20 $\mu \mathrm{M}$, $n=3$ ), as demonstrated previously in the hippocampus [7]. Following complete washout of APV, it was possible to induce LTP in these same neurons. However, in comparison to potentiated responses in the hippocampus that are not sensitive to APV [7], the amplitude of a potentiated I-EPSP was reduced or abolished by APV. This is not unexpected, given the APV-sensitive nature of the l-EPSP prior to induction of LTP.

Our results indicate that a novel l-EPSP can be evoked in regular-spiking neurons 
of layer II-III of the rat frontal cortex. This APV-sensitive l-EPSP appears to be generated by a polysynaptic circuit and displays LTP following tetanic stimulation. Stimulation intensities necessary to induce LTP in frontal cortex were quite low and often evoked no discharge of the impaled neuron. However, the LTP of I-EPSPS observed here is presumably a reflection of suprathreshold changes occurring in other cells in a polysynaptic circuit. Our induction of LTP with low stimulus intensities is consistent with the observation that hippocampal interneurons can show LTP at weak stimulus strengths that do not potentiate pyramidal cells [6].

Activation of NMDA receptors has been shown to be necessary for obtaining LTP in the rat visual cortex [2]. Under normal conditions, however, LTP was seen only in bursting neurons; potentiation could be obtained in regular-spiking cells only after blockade of GABAergic inhibition [2]. It was concluded that inhibitory pathways effectively control activation, in regular-spiking cells, of the NMDA-mediated processes giving rise to LTP. Our results indicate that intact inhibition does not prevent the induction of LTP but can mask its expression. Thus, LTP appears to be a general property of frontal cortex under normal conditions. The locus of control of LTP in the neocortex may not be exclusively pre- or postsynaptic but rather an emergent property of activity in a population of interconnected neurons. Similar plasticity in local synaptic circuits has been observed in the hippocampus [13].

This work was supported by National Institutes of Health Grants NS22373 and NS18145, by Deutsche Forschungsgemeinschaft Grant Su 104/1-1, and by a Fellowship Award from the Max Kade Foundation to B.S.

1 Andersen, P., Sundberg, S.H., Svenn, O. and Wigström, H., Specific long-lasting potentiation of synaptic transmission in hippocampal slices, Nature (Lond.), 266 (1977) 736-737.

2 Artola, A. and Singer, W., Long-term potentiation and NMDA receptors in rat visual cortex, Nature (Lond.), 330 (1987) 649-652.

3 Baranyi, A. and Feher, O., Conditioned changes of synaptic transmission in the motor cortex of the cat, Exp. Brain Res., 33 (1978) 283298.

4 Berry, M.S. and Pentreath. V.W., Criteria for distinguishing between monosynaptic and polysynaptic transmission, Brain Res., 105 (1976) 1-20.

5 Bindman, L.J., Lippold, O.C.J. and Milne, A.R., Prolonged changes in excitability of pyramidal tract neurones in the cat: a postsynaptic mechanism, J. Physiol. (Lond.), 286 (1979) 457-477.

6 Buzsaki, G. and Eidelberg, E.. Direct afferent excitation and long-term potentiation of hippocampal interneurons, J. Neurophysiol., 48 ( I 982) 597 607.

7 Collingridge, G.L., Kehl, S.J. and McLennan, H., Excitatory amino acids in synaptic transmission in the Schaffer collateral commissural pathway of the rat hippocampus, J. Physiol. (Lond.), 334 (1983) $33-46$.

8 Flatman, J.A., Schwindt, P.C. and Crill, W.E. The induction and modification of voltage-sensitive responses in cat neocortical neurons by N-methyl-D-aspartate, Brain Res., 363 (1986) 62-77.

9 Howe, J.R., Sutor, B. and Zieglgänsberger, W., Characteristics of long-duration inhibitory postsynaptic potentials in rat neocortical neurons in vitro, Cell. Mol. Neurobiol., 7 (1987) $1-18$.

10 Jordan, W., Sutor, B. and Zieglgänsberger, W., The action of L-glutamate, L-aspartate and $\mathrm{N}$-methylD-aspartate (NMDA) on membrane potential and input resistance of rat neocortical neurons in vitro, Pflügers Arch., 405 Suppl. 2 (1985) R45.

11 Lømo, T., Potentiation of monosynaptic EPSPs in the perforant path-dentate granule cell synapse, Exp. Brain Res., 12 (1971) 4663. 
12 McCormick, D.A., Connors, B.W., Lighthall, J.W. and Prince, D.A., Comparative electrophysiology of pyramidal and sparsely spiny stellate neurons of the neocortex. J. Neurophysiol., 54 (1985) 782-806.

13 Miles, R. and Wong, R.K.S., Latent synaptic pathways revealed after tetanic stimulation in the hippocampus, Nature (Lond.), 329 (1987) 724726.

14 Pellionisz, A. and Llinas, R., Space-time representation in the brain. The cerebellum as a predictive space-time metric tensor, Neuroscience, 7 (1982) 2949-2970.

15 Peters, A.. Synaptic specificity in the cerebral cortex. In G.M. Edelman, W.E. Gall and W.M. Cowan (Eds.). Synaptic Function, Wiley, New York, 1987, pp. 373397.

16 Sutor, B., Jordan, W, and Zieglgänsberger. W., Evidence for a magnesium-insensitive membrane resistance increase during NMDA-induced depolarizations in rat neocortical neurons in vitro. Neurosci. Lett., 75 (1987) 317. 322.

17 Thomson, A.M., A magnesium-sensitive post-synaptic potential in rat cerebral cortex resembles neuronal responses to N-methylaspartate, J. Physiol. (Lond.), 370 (1986) $531-549$.

18 Weiss, D.S. and Hablitz, J.J., Interaction of penicillin and pentobarbital with inhibitory synaptic mechanisms in neocortex, Cell. Mol. Neurobiol.. 4 (1984) 301317.

19 Wigstrom. H., Associative recall and formation of stable modes of activity in neural network models. J. Neurosci. Res., I (1975) 287.313.

20 Yamamoto, C. and Chujo, T., Long-term potentiation in thin hippocampal sections studied by intracellular and extracellular recordings, Exp. Neurol., 58 (1978) 242-250. 